\title{
Multilinguales
}

\section{Contexte et théorie de l'impolitesse dans quelques comédies de Molière}

Contextualization in a Plurilingual Milieu: The Example of Saint-Martin

Overseas Community

\section{Kais Amri}

\section{CpenEdition}

\section{Journals}

Édition électronique

URL : http://journals.openedition.org/multilinguales/2684

DOI : 10.4000 /multilinguales. 2684

ISSN : 2335-1853

Éditeur

Université Abderrahmane Mira - Bejaia

Édition imprimée

Date de publication : 1 décembre 2013

Pagination : 155-170

ISSN : 2335-1535

\section{Référence électronique}

Kais Amri, «Contexte et théorie de l'impolitesse dans quelques comédies de Molière », Multilinguales [En ligne], 2 | 2013, mis en ligne le 01 décembre 2013, consulté le 17 septembre 2019. URL : http:// journals.openedition.org/multilinguales/2684; DOI : 10.4000/multilinguales.2684

Ce document a été généré automatiquement le 17 septembre 2019.

\section{cc) (†)}

Multilinguales est mise à disposition selon les termes de la Licence Creative Commons Attribution -

Pas d'Utilisation Commerciale - Pas de Modification 4.0 International 


\title{
Contexte et théorie de l'impolitesse dans quelques comédies de Molière
}

\author{
Contextualization in a Plurilingual Milieu: The Example of Saint-Martin \\ Overseas Community
}

Kais Amri

1 Nous allons étudier des situations de confrontation des personnages dans quelques comédies de Molière, à travers l'ensemble des situations linguistiques et extralinguistiques qui favorisent et alimentent les conflits et les désaccords propices à l'émergence des phénomènes comme l'insulte, l'interruption, l'ordre et l'obligation. Dans le cadre de cet article nous allons nous focaliser sur le contexte parce qu'à notre avis, il est la pierre angulaire de tout l'édifice interprétatif des scènes de tension.

2 Nous allons commencer par fixer le cadre théorique de notre approche en référence à des théories pragmatiques comme le principe de coopération de Grice, la théorie de pertinence de Sperber et Wilson, mais également à des théories qui relèvent de la sociolinguistique comme les théories de la politesse élaborées par Brown, Levinson et Leech et surtout la théorie de l'impolitesse élaborée par des théoriciens comme Bousfield et Culpeper.

\section{Le cadre théorique}

\section{Le principe de coopération de Grice et la communication tendue}

3 Le principe de coopération (Grice, 1991 : p. 20) a été élaboré par Grice au cours de ses recherches sur la communication interindividuelle :

Nos échanges de parole ne se réduisent pas normalement à une suite de remarques décousues et ils ne seraient pas rationnels, si tel était le cas. Ils sont de manière caractéristique, jusqu'à un certain point au moins, le résultat d'efforts de coopération; et chaque participant reconnaît jusqu'à un certain point dans ces échanges un but commun ou (...) au moins une direction acceptés réciproquement. (Grice, 1979 : 13) 
4 Il a posé que tout acte d'échange verbal soutient une idée de coopération. C'est-à-dire que chaque pôle de l'échange fait en sorte que ses propos soient clairs et intelligibles pour son interlocuteur. L'échange "confrontationnel " ne déroge pas à cette règle générale de coopération. Ainsi, même si le locuteur transgresse volontairement l'une des quatre maximes ${ }^{79}$ sur lesquelles est fondé le principe de coopération, il peut tout de même réussir à véhiculer une information dont son interlocuteur serait capable d'en inférer le sens. Dans ce cas, le contexte se présente comme un élément indispensable à la bonne compréhension de l'énoncé proféré par un locuteur A au cours d'un échange $\mathrm{x}$.

5 Ces transgressions volontaires (ostensibles) sont appelées par Grice des « implicatures » conventionnelles ou conversationnelles. L'implicature conventionnelle est intimement liée au contexte linguistique de l'énoncé. Ainsi, un terme quelconque (verbe, adverbe, adjectif, préposition, etc.) peut changer ou du moins réguler la portée sémantique de tout l'énoncé. En ce qui concerne l'implicature conversationnelle, elle est directement liée au contexte situationnel dans l'élaboration d'un sens second autre que le sens littéral véhiculé par l'énoncé. Ainsi le terme « chien » n'est pas insultant en soi, mais utilisé dans un contexte situationnel d'insulte et réactivé par tout l'univers négatif de valeurs qui l'entoure, il le devient. Il peut être considéré dans un tel contexte comme une insulte des plus fortes.

\section{Théorie de pertinence et confrontation interindividuelle}

6 La théorie de pertinence s'est développée au sein même du courant pragmatique. (Moeschler, 2009: 178). À l'encontre du principe de coopération de Grice qui se présente comme un principe rationnel. (Moeschler, 2009: 178), la théorie de pertinence élaborée par Sperber et Wilson se définit, quant à elle, comme une approche cognitiviste $^{80}$ :

Rien n'oblige à accepter comme vraie la présomption de pertinence communiquée par un énoncé. Le communicateur peut ne pas avoir réussi à être pertinent; le destinataire peut douter de la capacité du communicateur à être pertinent. Mais la présomption de pertinence entraîne une présomption plus fiable, à savoir que le communicateur a essayé d'être pertinent, qu'il y soit parvenu ou non. (Sperber et Wilson, 1986 : pp. 238-239)

7 Pour Sperber et Wilson, les interlocuteurs ne cherchent pas, au cours d'un échange quelconque, à être coopératifs mais plutôt à être pertinents ${ }^{81}$. Toutefois, l'impertinence d'un énoncé peut être causée par divers facteurs. Elle peut être accidentelle ou voulue. Dans le cas où elle est accidentelle, elle peut révéler davantage d'informations sur la personnalité du locuteur (maladie, fatigue, troubles psychiques, etc.). En cas d'impertinence voulue et préméditée, elle reflète, entre autre, la volonté du locuteur de ne pas communiquer avec son interlocuteur. Le contexte intervient, dans le premier comme dans le deuxième cas, comme un élément déterminant pour distinguer un énoncé impertinent accidentel d'un énoncé impertinent calculé. Il fait découvrir également les intentions du locuteur telles que : fuir son interlocuteur, déjouer ses attentes, se moquer de lui en affichant à son égard une fausse attitude polie. De ce fait, un énoncé ne peut pas être jugé pertinent ou impertinent qu'en étant confronté à son contexte. Imaginons, à titre d'exemple l'échange suivant :

- A : «On est le combien aujourd'hui ?»

- B : «J'ai eu une mauvaise note en maths» 
La réponse de B peut s'averer, de prime abord, inpertinente. Mais le contexte immédiat n'explique pas tout, il faut se référer ici à un contexte plus large (contexte situationnel) pour décrypter le sens que $\mathrm{B}$ cherche à transmettre à $\mathrm{A}$, la relation qui unit $\mathrm{A}$ à $\mathrm{B}$ et le degré de partage des mêmes valeurs et des mêmes compétences.

Si B est étranger (il ne parle pas couramment la langue de A), A peut inférer que B n'a pas compris sa question parce qu'il ne maîtriserait pas assez sa langue. Il a le choix dans ce cas de reformuler sa question.

Si A et B sont deux collègues et qu'ils s'en veulent mutuellement (pour une cause ou pour une autre), A peut inférer que B a produit cet énoncé pour se moquer indirectement de lui et lui rappeler leurs années scolaires pendant lesquelles A a toujours été médiocre en mathématiques alors que maintenant il occupe un poste plus important que celui de B.

11 Si A est parent de B et qu'il est au courant qu'aujourd'hui le professeur de maths va rendre les copies aux élèves, nous nous trouvons face à une situation totalement différente $: B$ a décrypté le sens caché (implicite) de la question posée par son père ou sa mère et a répondu de façon directe à une question formulée indirectement.

Nous remarquons d'après cet exemple à quel point le contexte est indispensable à une interprétation correcte d'un énoncé.

13 Il faut mentionner également que ces mêmes scènes de confrontation peuvent être également étudiées à la lumière des théories sociolinguistiques.

\section{La confrontation communicationnelle à la lumière des théories sociolinguistiques}

La sociolinguistique est une approche qui essaye de recourir à des concepts qui relèvent du paradigme sociologique comme : face, territoir $e^{82}$, etc., pour comprendre tout ce qui relève de la communication interindividuelle. Elle se présente ainsi comme l'étude descriptive de l'effet de tous les aspects de la société, y compris les normes culturelles et le contexte, sur la façon dont la langue est utilisée. Elle s'intéresse également aux effets de l'utilisation de la langue sur la société.

Pour Goffman, chaque locuteur en situation de communication doit respecter un certain « rituel » communicationnel. Il s'efforce de :

(...) mobiliser les informations dont il dispose déjà à leur sujet à l'ouverture de l'interaction (informations dont la quantité et la qualité varient considérablement d'une interaction à l'autre), (...) chercher à extraire celles qui viennent à s'actualiser tout au long de son déroulement. (Goffman, 1973 : p. 11)

a - La politesse : la notion de politesse a été étudiée, tout d'abord, en profondeur par des sociolinguistes comme Brown et Levinson dans leur livre : Politeness : Some Universals in Language Usage (Brown et Levinson, 1987). Ils ont surtout affiné des notions comme la face, et ce en posant qu'en situation de communication entre deux individus, quatre faces entrent en jeu: les faces positive et négative du locuteur et les faces positive et négative de l'interlocuteur : 


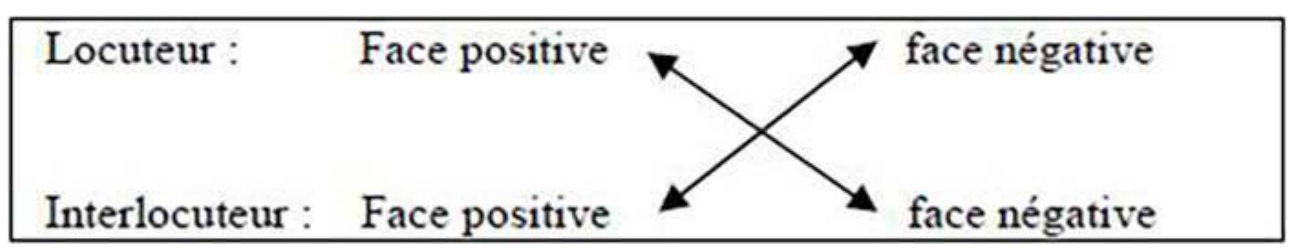

Toute personne en situation de communication doit prendre en considération, en plus de ses deux propres faces, les deux faces de son interlocuteur. En d'autres termes, tout système conversationnel dans lequel sont impliquées deux personnes, quatre faces entrent en jeu. Ces quatre faces seront menacées par un ensemble d'actes de langage produits par les interactants en question.

Ainsi en usant de la parole, chaque locuteur doit être attentif à ne pas blesser les faces de son interlocuteur, mais également à ne pas perdre les siennes. Produire un acte menaçant de la face de l'interlocuteur $F T A^{83}$ peut être atténué par la production d'un adoucisseur ou d'un $F F A^{84}$. Mais la profération même d'un FTA ou d'un FFA ne peut être appréhendée correctement qu'en se référant au contexte même de l'énoncé. Ainsi, l'impératif qui véhicule ordinairement un acte menaçant de la face positive (amour propre, estime de soi, etc.) ou négative (champ de liberté de l'interlocuteur, etc.) peut cesser de l'être dans un contexte d'urgence de demande d'aide («aidez-moi »), ou dans un contexte de stimulation publicitaire : « gagnez un million de dollars! ».

b - L'impolitesse : l'impolitesse serait pour des sociolinguistes tels que Brown et Levinson un fait parasitaire qui vient se greffer sur le phénomène englobant qu'est la politesse. Mais Bousfield et Culpeper ne sont pas du même avis. Ils considèrent que le méga-phénomène qui organise et balise la majorité des échanges interhumains relève de l'impolitesse; la politesse ne couvre qu'une partie minime de la communication. Ainsi tout geste, toute prise de parole sont des atteintes potentielles à la personne en face de nous. La politesse conventionnelle et sociale n'est que la partie superficielle et apparente de la personnalité humaine au cours de l'interaction. Toutefois, la partie submergée des conflits et des désaccords reste, dans la majorité des cas, enfouie et intentionnellement dissimulée par les locuteurs.

Donc, afficher sa politesse à l'égard de l'autre ne signifie pas toujours être sincère. La preuve en est que la politesse, elle-même, peut véhiculer une attitude impolie, comme dans le cas de l'ironie. Elle est aussi intimement liée au contexte de l'échange et à l'importance de l'axe vertical et de l'axe horizontal qui séparent A de B. (Moeschler, 2009: 197). Pour mieux étudier les relations interpersonnelles qui régissent et influencent les interactions verbales, Brown et Levinson ont forgé un certain nombre d'autres concepts. Ainsi le locuteur, pour ne pas blesser la face de son interlocuteur ni sa propre face, doit prendre en considération deux facteurs qui sont: "la force sociale " (the social power), qui représente le pouvoir du locuteur sur l'interlocuteur et qui est l'équivalent de l'axe vertical chez Moeschler, et « la distance sociale » (the social distance), symbolisée par la lettre (D) et qui équivaut à l'axe horizontal.

21 Nous allons consacrer la deuxième partie de cet article à l'analyse de situations «confrontationnelles» dans quelques comédies de Molière ${ }^{85}$, à travers deux phénomènes : l'insulte et l'interruption. 


\section{L'insulte}

L'insulte dans le théâtre de Molière prend, à notre sens, deux formes principales: insulter en utilisant un évaluatif axiologique négatif comme les adjectifs qualificatifs (lâche, traître, pendard, etc.), les noms abstraits à connotation négative (sottise, bêtise, etc.), et surtout les noms d'animaux (métaphore lexicalisée) pour qualifier les hommes (chien, serpent, crocodile, âne, bœuf, étourneau, mirmidon, vers, etc.).

23 Nous allons essayer de répertorier ces insultes tout d'abord selon leur nature grammaticale (nom, adjectif, etc.), ensuite selon les relations sémantiques qu'elles entretiennent les unes avec les autres: rapport d'hyperonymie-hyponymie qui relie, par exemple, bête à chien, animal à crocodile, etc.

\section{« Bête » et « animal »}

Le premier exemple, extrait de la pièce La Jalousie du Barbouillé (Molière, 1673 : I-1), nous peint l'attitude verbale du Barbouillé envers sa femme Angélique (Molière, 1673 : I-1) : il la qualifie de "bête » et la menace de violence physique; mais il ne s'adresse pas directement à elle puisque la réplique se fait en aparté et prend donc la forme de A dit à A que C. L'information est présentée également à $(D a)^{86}$ :

Si je l'eusse trouvée en vie, après m'avoir fait cette frayeur-là, je lui aurais apostrophé cinq ou six clystères de coups de pied dans le cul parce qu'elle a fait la bête en cherchant à se donner la mort. (Molière : $1673: \mathrm{I}-1$ )

Le Barbouillé la traite de "bête ", c'est-à-dire qu'il la qualifie de personne sotte et dénuée de raison parce qu'elle cherche à se suicider. Le suicide est donc vu dans ce contexte comme un acte contre le bon sens. Toutefois l'insulte «bête » est adoucie par sa forme même, puisqu'il ne s'agit pas d'une insulte directe, à la face de l'interlocuteur B (Culpeper, 2011 : p. 32). Il s'agit au contraire d'un propos que A adresse à lui-même et qu'il confie en même temps à $(D a)$, témoin privilégié de l'énonciation théâtrale. (Ubersfeld, $1996:$ p. 24). Mais, cette insulte «bête» représente une atteinte à la face positive de B (même indirectement) puisqu'elle tente de blesser l'amour propre et l'estime de soi de B.

Dans le second exemple, extrait de la pièce L'Etourdi, l'emploi du terme «bête » prend la forme d'une interjection utilisée par Mascarille pour exprimer son indignation face au comportement sot et insensé de son maître Lélie: "La peste soit la bête.». (Molière, 1663 : I.4.)

Dans ce contexte également la force illocutionnaire (Searle, $2008:$ p. 105) de l'énoncé est épargnée par la forme indirecte de l'insulte puisqu'il s'agit également ici d'un aparté de forme $\mathrm{A}$ dit à $\mathrm{A}$ que $\mathrm{C}$ en prenant $(\mathrm{Da})$ pour témoin. Le terme «bête " utilisé comme insulte sert d'attaque indirecte à la face négative de l'interlocuteur en s'attaquant à ses besoins de face d'être estimé, respecté et aimé selon la théorie de Brown et Levinson (Brown et Levinson, 1987 : p. 65). Rabaisser l'homme au rang de bête, c'est le priver de facultés humaines comme la raison, la conscience, etc.

L'analogie entre le comportement de l'homme et celui de l'animal ou de la bête a fait l'objet de plusieurs tentatives d'explications.

Aristote dans son Histoire des animaux décrit l'homme comme un animal (Aristote, 1883 :

livre X : 25), mais un animal raisonnable, doté de raison et de conscience. Montaigne, 
dans l'apologie de Raimond Sebond, relève que d'une manière générale "la manière de naître, d'engendrer, vivre et mourir des bestes est si voisine de la nostre " (Montaigne, 1979 : vol 2, p. 83). raison, de conscience. La force illocutionnaire de cette insulte peut être intensifiée par 
l'adjonction d'adjectifs et causer plus de dommage aux besoins de face de l'insulté. Examinons les exemples suivants :

- Premier exemple extrait de la pièce L'Etourdi :

Anselme : «Ce fier animal pour toutes les prières, Ne perdrait pas un coup de ses

dents meurtrières, Tout le monde y passe. » (Molière, 1663 : VI-3)

- Second exemple extrait de la pièce L'Impromptu de Versailles :

Molière : " $\mathrm{Ah}$ ! Les étranges animaux à conduire que des comédiens. » (Molière, $1663: \mathrm{I}-1)$.

- Troisième exemple extrait de la pièce Le Tartuffe, ou l'imposteur : Orgon : «L'homme est, je vous l'avoue, un méchant animal ! (Molière, 1669 : V- 5)

Les adjectifs «fier » (exemple $n^{\circ} 1$ ), «étranges » (exemple $\left.n^{\circ} 2\right)$, « méchant » (exemple $n^{\circ}$ 3), qui déterminent le substantif « animal »/ »animaux », modifient son sens littéral. Ainsi dans l'exemple $n^{\circ} 1$, Anselme pensant que son ami Pandolfe est mort, s'emporte contre la mort en la qualifiant de «fier animal». Dans ce contexte la force illocutionnaire de l'insulte est épargnée par la nature abstraite de la cible de l'insulte (la mort).

41 De même la force illocutionnaire de l'insulte est diminuée par l'emploi d'un terme négatif (la mort); on ne peut pas penser que ce type d'insulte puisse provoquer une réaction $\mathrm{R}$ parce que l'insulté est abstrait. L'insulte prend donc la forme de $A$ dit à $B$ que $\alpha$.

Dans l'exemple $n^{\circ} 2$, Molière qualifie tous les comédiens d'« étranges animaux », dans un contexte ironique. Il se moque d'eux parce qu'ils ne maîtriseraient pas leurs rôles. Il répond ainsi à Brécourt qui s'inquiète du peu de temps dont ces comédiens disposent pour cela: "Que voulez-vous qu'on fasse? Nous ne savons pas nos rôles; et c'est nous faire enrager vous-même, que de nous obliger à jouer de la sorte. » (Molière, 1663 : I-1).

La réaction $\mathrm{R}_{1}$ de Molière est une insulte verbale, « étranges animaux ", qui s'adresse à tous les comédiens, y compris Brécourt. L'adjectif qualificatif «étrange - $\mathrm{s}$ », du latin extraneux qui signifie "contraire à l'usage, à l'ordre, à l'habitude; bizarre, extraordinaire. $\|^{88}$, sert à qualifier les " animaux » auxquels sont comparés « les comédiens » qui seraient donc des « animaux » bizarres, extraordinaires, qui agissent de façon inhabituelle.

Ainsi, à l'assimilation des "comédiens" à des "animaux" les prive de leur «humanité » (le bon sens, la raison, la conscience, etc.), en les rabaissant au rang d'animal, s'ajoute un trait inhérent à cet animal (/étrange/) qui fait des comédiens une curiosité, des spécimens bizarres, qui échappent au processus linguistique habituel de catégorisation. L'adjectif qualificatif renforce donc la visée ironique de l'énoncé. L'insulte prend la forme de $A$ dit à $B$ que $B+C+\ldots$ sont non seulement des " animaux ", mais aussi d'« étranges animaux ».

Dans l'exemple $n^{\circ} 3$, l'animalité de l'homme se double de méchanceté. Par l'emploi générique du terme "homme ", Orgon, dans son entretien avec Valère et Cléante, fait allusion à Tartuffe qu'il qualifie donc «indirectement» de "méchant animal ». L'insulte prend donc la forme de $A$ dit à $B$ que $C$ est un « méchant animal ». La puissance de nuisance de Tartuffe, l'imposteur, est accrue par l'association de "son " animalité avec «sa » méchanceté. L'insulte s'attaque dans ce contexte à la face négative de $C$, c'est-à-dire à son estime de soi et à son amour propre. L'adjectif «méchant » sert à intensifier la force illocutionnaire de l'insulte. 


\title{
L'interruption
}

L'interruption agit comme une forme d'impolitesse quand un locuteur $\mathrm{B}$ cherche à bloquer la parole d' un locuteur $\mathrm{A}$, en changeant de sujet de la conversation, en dirigeant la conversation dans une autre direction ou en cherchant tout simplement à prendre congé de son interlocuteur. L'interruption peut s'effectuer également par l'intervention d'un tiers $(C)$ qui interrompt l'échange entre $A$ et $B$ en adressant la parole à $B$. Nous allons voir, à travers des exemples, le fonctionnement de l'interruption, à la lumière de la théorie de la politesse de Leech et la théorie de la face de Brown et Levinson.

Notre objectif est de déterminer le mécanisme du fonctionnement de l'interruption considérée comme une forme d'impolitesse, autrement dit comme une infraction au principe de politesse (PP ${ }^{89}$ de Leech et comme des FTA selon la théorie de Brown et Levinson et ce à travers des exemples extraits de la pièce La Jalousie du barbouillé.

\section{Forme d'interruption : $\mathrm{B}$ coupe la parole à $\mathrm{A}$}

\author{
- Le Barbouillé \\ A - Je vous prends pour un docteur. Or çà, parlons un peu de l'affaire que je vous \\ veux proposer ; il faut que vous sachiez... \\ - Le Docteur \\ B - Sache auparavant que je ne suis pas seulement une fois docteur, mais que je suis \\ une, deux, trois, quatre, cinq, six, sept, huit, neuf, et dix fois docteur : parce que, \\ comme l'unité est la base, le fondement et le premier de tous les nombres, aussi, \\ moi, je suis le premier de tous les docteurs, le docte des doctes ; parce qu'il y a deux \\ facultés nécessaires pour la parfaite connaissance de toutes choses, le sens et \\ l'entendement; et comme je suis tout sens et tout entendement, je suis deux fois \\ docteur. \\ - Le Barbouillé \\ A - D'accord. C'est que... \\ - Le Docteur \\ B - Parce que le nombre de trois est celui de la perfection, selon Aristote ; et comme \\ je suis parfait, et que toutes mes productions le sont aussi, je suis trois fois docteur. \\ (Molière, 1673 : I-2)
}

Nous avons déjà signalé que le principe général de Leech dit rhétorique interpersonnelle $(I R)^{90}$ repose sur deux principes : le principe de coopération de Grice, selon lequel la coopération est l'objectif de toute communication, et le principe de politesse (PP) de Leech composé de six maximes (tact, générosité, approbation, modestie, compromis, sympathie).

Ces maximes fonctionnent selon une loi bipolaire de coût/ bénéfice. Ainsi le locuteur peut diminuer le coût de l'énoncé adressé à $\mathrm{L}^{91}$ ou augmenter les bénéfices pour luimême. Ainsi, la maxime de tact, il faut, du point de vue de la politesse positive, « réduire le coût de l'acte pour les autres» (Leech, 1983 : 104) et, du point de vue de la politesse négative, "augmenter les bénéfices des autres " (ibid.: 104): "there is a tendency to exaggerate agreement with other people, and to mitigate disagreement by expressing regret, partial agreement, etc. » (Leech, 1983 : p. 138).

Il est évident que l'interruption, en tant que forme de subtilisation de la parole de $A$, fonctionne comme une infraction au principe de coopération de Grice. L'appropriation de la parole agit donc comme une déclaration de non coopération avec A; cet acte 
implique que les propos de $A$ n'ont pas l'importance requise pour qu'ils soient écoutés jusqu'à la fin, sans interruption. Le (Da) peut en déduire que $B$ n'est pas coopératif et que les propos de A sont dépourvus de l'intérêt requis. L'inférence peut être également faite au niveau de la relation qui unit $A$ à $B$. $B$ se donne le droit d'interrompre $A$ parce qu'il détient (ou pense détenir) le droit et l'habileté de le faire.

51 Ainsi le docteur se permet d'interrompre son interlocuteur (le Barbouillé) parce qu'il est convaincu qu'il détient un savoir qui l'autorise à interrompre quiconque; il se présente donc comme une sorte d'autorité morale et encyclopédique investie du droit de parler et d'interrompre. Par la façon d'agir de $B,(D a)$ peut faire également une inférence sur le comportement du Docteur qui serait un personnage pédant, volubile, qui cherche par tous les moyens à étaler sa compétence.

De point de vue du principe de politesse (PP) de Leech, nous observons que l'interruption, par son caractère abrupt et non coopératif, œuvre à «bafouer » toutes les recommandations des six maximes. Ainsi, du point de vue de la maxime de tact, telle qu'explicitée plus haut, $B$, en coupant la parole à quelqu'un, en lui confisquant son tour de parole, ne cherche réellement qu'à augmenter le coût de l'acte pour A parce que $(D p)$ et $(D a)^{92}$ peuvent considérer qu'il s'agit d'un comportement impoli de la part de $B$.

Dans cet exemple, le coût de l'acte augmenté par l'interruption est doublé par l'impératif ("sache auparavant que je ne suis pas seulement une fois docteur »), lequel, utilisé en association avec le tutoiement, agit également à augmenter le coût illocutionnaire pour $\mathrm{B}$ et à réduire ses bénéfices, d'où l'absence de toute sorte d'adoucisseurs et d'acte de langage indirects, conditionnel de politesse, vouvoiement, etc.

L'interruption peut également constituer une infraction de la maxime de générosité qui dicte, du côté de la politesse positive, de "minimiser les bénéfices pour soi-même » (Leech, 1983 : 133), et du côté de la politesse négative, d' «augmenter le coût pour soi-même» (ibid. : 133). Or $B$, dans le cadre du dialogue avec $A$, agit de façon tout à fait contraire à ces recommandations: le docteur s'est efforcé d'augmenter les bénéfices pour luimême (en étalant son savoir) et à diminuer le coût pour lui-même (en se flattant et en s'efforçant de s'approprier l'image du grand savant "parfait». Dans ce cas, (Da) peut inférer que c'est un personnage "anormal ", qui souffre très probablement d'une « hypertrophie du moi » et que sa présence répond à des fins comiques. L'exagération et la répétition justifient l'inférence faite par $(D a)$ à propos de $B$.

La maxime d'approbation (sous ses deux faces de politesse positive et de politesse négative) consiste à minimiser les blâmes d'autrui (politesse positive) et à augmenter l'éloge des autres (face négative). Or il semble, encore une fois, que le comportement de $A$ va à l'encontre de toutes ces recommandations : le docteur cherche à « maximiser les blâmes portés à autrui » et à " minimiser, voire annuler, l'éloge des autres ", de A en l'occurrence. La " maximisation » des blâmes portés à autrui se concrétise à travers la comparaison implicite de $B$ à $A$. Le docteur se présente comme le détenteur d'un savoir d'autant plus encyclopédique qu'il est face à un profane/ignorant $A$ qui sollicite son avis.

\section{Ordre et obligation}

Nous allons maintenant considérer l'ordre et l'obligation, toujours à travers le théâtre de Molière, à la lumière de la théorie des faces de Brown et Levinson : 
Premier exemple tiré de L'école des femmes:

\section{- Arnolphe à Agnès}

Je n'ai qu'un mot à dire, et ne tarderai guère.

Rentrez, et conservez ce livre chèrement.

Si le Notaire vient, qu'il m'attende un moment. (Molière, 1663 : vers 807)

Arnolphe poursuit son échange avec Agnès (B) en lui assignant l'ordre de rentrer et de conserver précieusement le «livre » des maximes de la bonne conduite. Du point de vue du $P C$ gricien, un tel comportement est jugé non coopératif et représente donc une éventuelle infraction au principe de coopération.

Si nous considérons maintenant cet impératif du point de vue de la théorie des faces de Brown et Levinson, nous remarquons que ce même ordre représente un FTA, Acte menaçant pour la face de l'interlocuteur, Agnès en l'occurrence. En proférant cet ordre, Arnolphe a bien commis un acte menaçant à l'égard de la face négative de $B$ et ce en limitant son champ de liberté, en s'immisçant dans son territoire, pour lui imposer sa volonté et son univers de croyance ${ }^{93}$. Comme signalé plus haut, la face négative de $B$ est menacée quand $A$ ne respecte pas la liberté de son interlocuteur $B$.

Examinons de plus près cet ordre pour mieux comprendre son fonctionnement. Il s'agit d'un ordre exprimé à travers un impératif et qui s'adresse à l'interlocuteur $B$ (Agnès), désigné par le pronom personnel vous. Le vouvoiement est généralement utilisé pour marquer une certaine relation de respect, de courtoisie, une distance entre le locuteur et l'interlocuteur. Or, ici, le recours au vouvoiement ne semble pas marquer une intention de politesse ou de respect vis-à-vis d'Agnès; au contraire, il contribue à renforcer la relation hiérarchique qui lie Arnolphe à Agnès, une relation de tuteur à pupille. Nous pouvons donc dire que ce vouvoiement n'est pas une marque d'adoucissement d'un ordre trop direct qui aurait demandé d'être atténué pour être socialement acceptable; il sert plutôt à déterminer la relation « verticale » qu'Arnolphe entretient avec Agnès.

\section{Agression et pouvoir}

61 Les deux types d'agression, verbale et physique, sont considérés comme des actes qui menacent l'une ou l'autre face des interlocuteurs. La définition de la violence peut être restreinte à « la violence verbale » (l'insulte, l'injure, les refus, l'antipathie, etc.), ou élargie à " la violence physique ». Il existe toujours une cause plus au moins directe qui pousse $\mathrm{A}$ à agir de manière jugée violente à l'égard de $B$.

Des chercheurs en psychologie du développement considèrent l'agression comme un éventuel résultat d'une intelligence sociale qui manque d'empathie. (Björkqvist et al., 2000 : pp. 191-200). Ils notent qu'il y a plusieurs types de violence : de la forme la plus directe, comme l'agression physique, aux formes « intermédiaires », comme l'agression verbale directe (injure, menace, insulte, etc.), et les formes les moins directes (l'ironie, la fausse politesse, etc. ).

Berkowitz définit l'agression comme un comportement destiné à blesser quelqu'un physiquement ou psychologiquement (Berkowitz, 1993).

Spielberger et al. ont analysé un syndrome, qu'ils ont baptisé l'A.H.A syndrom ${ }^{94}$ « anger, hostility and agressivity " (Spielberger et al, 1985 : pp. 159-178) que l'on peut traduire par 
C.H.A : «Colère, hostilité, agressivité ». Ils ramènent les causes de toute agression (verbale et autre) à ces trois phénomènes psychologiques.

Ainsi tout comportement «impoli » procède d'un ensemble de facteurs personnels (énonciation) et contextuels (contexte de l'énonciation). Ainsi la colère peut être à l'origine de l'impolitesse, mais la cause qui provoque la colère peut varier d'une personne à une autre et d'un temps à un autre (Jay, 1992 : p. 98).

Plusieurs paramètres contribuent à contextualiser l'impolitesse : qui parle (l'identité de A), il parle à qui (l'identité de B), il parle de quoi (le sujet), pourquoi (l'objectif), où (cadre spatial), quand (cadre temporel).

Nous concluons donc que pour mieux comprendre la portée sémantique d'un énoncé agressif, il faut se référer au contexte, indispensable à son interprétation. C'est ce que nous avons tenté d'étudier, dans le cadre de cet article, en nous focalisant sur la relation des situations de confrontations avec leurs contextes d'énonciation.

\section{BIBLIOGRAPHIE}

ARISTOTE, Histoire des animaux, Librairie Hachette et Cie, Paris, 1883.

BOUSFIELD Derek, Impoliteness in interaction, Amsterdam/Philadelphia: John Benjamins Publishing Company, 2008.

BERKOWITZ Leonard, Agression: Its Causes, Consequences and Control, Philadelphia: Temple University Press, 1993.

BJÖRKQVIST Kaj, Karin ÖSTERMAN, et Ari KAUKIAINEN, « Social Intelligence - Empathy= Agression? », in Agression and Violent behaviour, $\mathrm{n}^{\circ}$ 5, 2000, pp. 191-200.

BROWN Penelope, Stephen LEVINSON, Politeness: Some Universals in Language Usage, Cambridge University Press, 1987.

CULPEPER Jonathan, Impoliteness: using language to cause offense, Cambridge University Press, 2011.

FARET Nicolas, l'Honnête homme ou l'Art de plaire à la cour, De l'incommodité que donnent les grands parleurs, Slatkine Reprints, 1970.

GOFFMAN Erving, La mise en scène de la vie quotidienne, Paris, Minuit, 1973.

GRICE Herbert Paul, Studies in the Way of Words, Logic and conversation, First Harvard University Press paperback edition, 1991.

JAY William, Cursing in America: a psycholinguistic study of dirty language in the courts, in the movies, in the schoolyards and on the street, John Benjamins, 1992.

KERBRAT-ORECCHIONI Catherine, Le discours en interaction, Armand Colin, 2005.

LEECH Geoffrey, Principle of Pragmatics, Longman, 1983.

MOESCHLER Jacques, Introduction à la linguistique contemporaine, Armand Colin, 2009. 
MOLIÈRE : corpus consulté sur la base informatisée FRANTEXT :

- L'Étourdi ou les Contre-temps, 1663, http://www.atilf.fr/

- Dépit amoureux, 1663, consulté sur le site http://www.atilf.fr/

- L'École des femmes, 1663, consulté sur le site http://www.atilf.fr/

- L'Impromptu de Versailles, 1663, consulté sur le site http://www.atilf.fr/

- Le Tartuffe ou l'Imposteur, 1669, consulté sur le site http://www.atilf.fr/

- La Jalousie du barbouillé, 1673, consulté sur le site http://www.atilf.fr/

MONTAIGNE, Essai, vol. 2, Flammarion, 1979.

SEARLE John R., Les actes de langage, Hermann, 2008.

SPIElBERGER, C. D., E. H. JOHNSON, S. F. RusSEll, R. CRANe, G. A. JACOBS, \& T. J. WORDEN. “The experience and expression of anger: Construction and validation of an anger expression scale", in Anger and hostility in cardiovascular and behavioural disorders, N.A.Chesney and R.H. Rosenman (eds.), 1985, New York: McGraw-Hill, pp. 159-187.

SPERBER Dan, Deirdre WILSON, Relevance : Communication and Cognition, Wiley, 1995.

UBERSFELD Anne, Lire le théâtre, vol. 1, Belin, 1996.

YLLÖ Kersti, « Through a Feminist Lens: Gender, Power and Violence », Édité par R. Gelles and D. Loseke (eds.), dans Controversies in Family Violence (Thousand Oaks, London, New Delhi: Sage Publications), 1993, pp. 47-62

\section{NOTES}

79. Maximes de quantité, de qualité, de relation et de manière.

80. La Théorie de la pertinence repose sur les fondements théoriques dans l'hypothèse cognitive qu'est la théorie de la modularité de l'esprit de Jerry Fodor et la grammaire générative de Noam Chomsky.

81. Fournir une énoncé qui ne soit pas décousu et ce par le minimum d'effort et le maximum d'effet cognitifs.

82. Ces notions (face, territoire, désir de face...etc.) ont été tout d'abord élaborées par Erving Goffman dans son livre: La Mise en scène de la vie quotidienne. (1979), reprisés, développées et affinées par Brown et Levinson.

83. Face Threatening Act est généralement traduit par : « Acte menaçant de la face ».

84. Face Flattering Act peut être traduit par « acte valorisant ou flatteur de la face ».

85. L'Étourdi ou les Contre-temps (1663), Le Dépit amoureux (1663), L'École des femmes (1663), L'Impromptu de Versailles (1663), Le Tartuffe ou l'Imposteur (1669), La Jalousie du barbouillé (1673).

86. « $D a »$ est le destinataire-audience ou le public dans le cas de la représentation théâtrale.

87. Selon Bousfield dans son livre Impoliteness in interaction, la réaction $\mathrm{R}$ de $\mathrm{B}$ à l'acte d'impolitesse commis par A peut prendre plusieurs formes : silence, riposte défensive, riposte offensive, violence physique, fuite.

88. Définition extraite du Petit Larousse encyclopédique, Librairie Larousse, 1980.

89. Principe de politesse.

90. Interpersonal rhetoric.

91. Locuteur.

92. $(D p)$ est le destinataire-personnage ; $(D a)$ est le destinataire-audience.

93. Dans ce contexte, Arnolphe croit qu'il a raison et qu'il détient le plein droit de guider Agnès, voire de la manipuler. 
94. AHA syndrom = syndrom of Anger, Hostility and Aggressivity : Syndrome de Colère, Hostilité et Agressivité (traduit par nous).

\section{RÉSUMÉS}

Toute relation interhumaine suppose une communication possible. Mais communication ne signifie pas toujours entente. Elle peut parfois manifester un comportement hostile, extériorisé à travers des phénomènes verbaux et langagiers comme l'insulte et l'interruption, l'ordre et l'obligation. Dans ce cas, le contexte fonctionne comme la clef de voûte pour une compréhension plus approfondie de l'énoncé proféré. En ne se référant pas au contexte, le sens de l'énoncé reste l'objet d'interprétations divergentes voire contradictoires. Subséquemment et pour mieux comprendre la portée, les objectifs et les finalités des insultes échangées et des interruptions pratiquées dans les comédies de Molière, il faut avoir une vue d'ensemble des déroulements des événements et des relations entre les différents personnages. C'est le sujet de notre étude.

Saint Martin, a French-Dutch Caribbean island, is characterized by a situation of multilingualism (French, English, Spanish, Creole). Needless to say, in such a context, the issue of educational and teaching strategies comes up to the front. The high schools from the French side struggle to implement France's educational reforms and especially the "New High School" measures from 2010, particularly personalized support. This study aims to establish as clearly as possible how this measure (personalized support) can support a dynamic and relational teaching more specific to Saint-Martin. To achieve our aim, we will take as example, two corpora (French/English), resulting from observation of exchanges between groups engaged in personalized support.

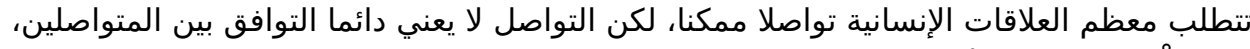

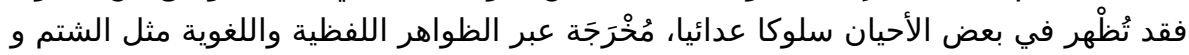

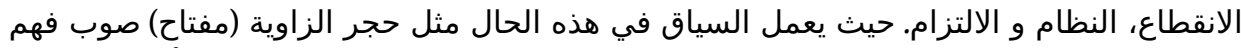

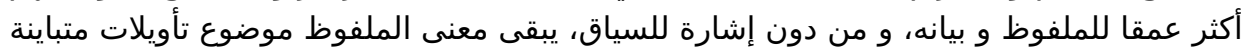

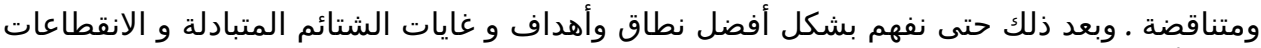

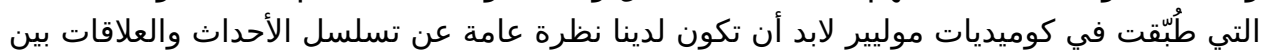

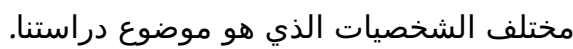

\section{INDEX}

مبدأ التعاون, السياق, نظرية الحصافة ( الوجاهة), نظرية التأدب, نظريةفهرس الكلمات المفتاحية:

Mots-clés : principe de coopération, contexte, théorie de la pertinence, théorie de la politesse, théorie de l'impolitesse

Keywords : teaching action, code-switching, school context, interactions, multilingualism 
AUTEUR

KAIS AMRI

Université de Clermont-Ferrand - EA 1002 CELIS - France 